\title{
Prevalence of Sarcocystis infection in processed meat products by using digestion and impression smear methods in Hamedan, Iran
}

\author{
Zainab Sadeghi Dehkordi ${ }^{1} \cdot$ Banafsheh Yalameha $^{2} \cdot$ Abbas Ali Sari $^{3}$
}

Received: 12 September 2016 / Accepted: 25 April 2017 / Published online: 9 May 2017

(C) The Author(s) 2017. This article is an open access publication

\begin{abstract}
Sarcocystis is a common zoonotic parasite which can be transmitted through ingestion of contaminated, undercooked meat and is a major cause of economic loss in many countries. This study aimed to detect Sarcocystis parasite in processed meat products in Hamedan, Iran. A total of 20 samples of hamburger, sausage, and cocktail were collected from markets from three factories in Hamedan, Iran. The samples were examined by digestion and impression smear methods for detecting Sarcocystis parasite. The results showed that $80 \%$ of all tested samples were infected with Sarcocystis. The infection rate in hamburger, sausage, and cocktail were 87.5, 83.33, and $66.66 \%$, respectively. The highest infestation rate was observed in hamburger. The present study shows that the rate of Sarcocystis contamination in meat products is very high. So, evaluation of raw meat quality in addition to applying hygienic programs at all stages of the production line is inevitable. Also, consumption of undercooked meat products or fast food should be avoided.
\end{abstract}

Keywords Sarcocystis $\cdot$ Processed meat-products $\cdot$ Hamedan

Zainab Sadeghi Dehkordi

dehkordisz@gmail.com

1 Department of Parasitology, Faculty of Para-Veterinary Science, Bu-Ali Sina University, Hamedan, Iran

2 Laboratory Science, Faculty of Para-Veterinary Science, Bu-Ali Sina University, Hamedan, Iran

3 Department of Food Hygiene and Quality Control, Faculty of Para-Veterinary Science, Bu-Ali Sina University, Hamedan, Iran

\section{Introduction}

The genus Sarcocystis includes more than 100 species with worldwide distribution. They are protozoan coccidian parasites belonging to the phylum Apicomplexa (Fayer 2004). Sarcocystis spp. are common parasites of a broad range of vertebrates, including mammals, birds, and fish. Merogony and cyst formation (asexual stage) take place in the intermediate host while gametogony and sporogony (sexual stages) take place in the definitive host. Human as both an intermediate and definitive host is more considerable in the life cycle of this parasite. Most pathogenic Sarcocystis spp. like $S$. humanis and S. suihumanis can cause infection in human as intermediate host (Fukuyo et al. 2002). The pathogenicity of Sarcocystosis spp. for human is uncertain. In most cases, they are not harmful or may be the cause of mild and transient gastrointestinal signs (Gabriele et al. 2006). The importance of muscular Sarcocystosis in farm animals is a well-documented problem. High prevalence of Sarcocystis infection is seen in cattle, pig, and sheep in both developing and industrialized countries (Sahl Poulsen and Rune 2014). Sarcocystosis in heavily infected animals causes reduced milk production, spontaneous abortions, and death (Fayer 2004). Infected animals can transmit the infection to other hosts via the fecal-oral transmission and leads to nausea, vomiting, and enteritis (acute, chronic, and severe) in human (Fayer et al. 2015). Also, human infection with Sarcocystis can be related to ingestion or water contamination with oocysts excreted by carnivorous definitive hosts or eating raw/undercooked meat containing the encysted parasite (Rosenthal et al. 2012). Consumption of processed meat products and fast foods are popular in all over the world (Mehdizadeh et al. 2014). Gabriele et al. found that the prevalence of Sarcocystis spp. in Argentinean beef was $33.4 \%$. In Iran, some studies were 
performed about Sarcocystis infection in meat products, for example in the study of Rahdar and Salehi (2011a, b), the rate of Sarcocystis infection in hamburger, hotdog, and sausage were seen 56,20 , and $8 \%$, respectively (Rahdar and Salehi 2011b). Up to now, in Hamedan, there is no study about the identification of Sarcocystis in meat products. Thus, the aim of this study was to investigate the Sarcocystis infection rate in processed meat products in Hamedan,

\section{Methods}

This study was carried out from October 2014 to March 2015; a total of 20 samples, including 8 hamburgers, 6 sausages, and 6 cocktails were collected from 3 plant (A, B, and C) products marketed in Hamadan, Iran and examined by digestion and impression smear (DOB smear) methods. In impression smear method, $2 \mathrm{~g}$ of each sample was impressed on the slide and fixed with methanol (70\%). All samples were stained by Giemsa staining method and observed microscopically for detecting Sarcocystis bradyzoite (Nourollahi Fard et al. 2009). In another method, each sample was digested by both following procedures:

Four grams of each sample $+3.5 \mathrm{~mL} \mathrm{HCl}$

$+2.5 \mathrm{~g}$ trypsin $+1500 \mathrm{~mL}$ distilled water

Two grams of each sample $+2.5 \mathrm{~g}$ trypsin

$+1500 \mathrm{~mL}$ distilled water

After digestion, all samples were incubated at $37{ }^{\circ} \mathrm{C}$ for $30 \mathrm{~min}$ and sieved through mesh and centrifuged at $2000 \mathrm{rpm}$ for $5 \mathrm{~min}$. The supernatant fluid was discarded and sediment was stained by Giemsa staining method. For Sarcocystis bradyzoite detection, all samples were examined by light microscope (40 and 100 magnification). Sarcocystis bradyzoite were shown in Figs. 1 and 2.

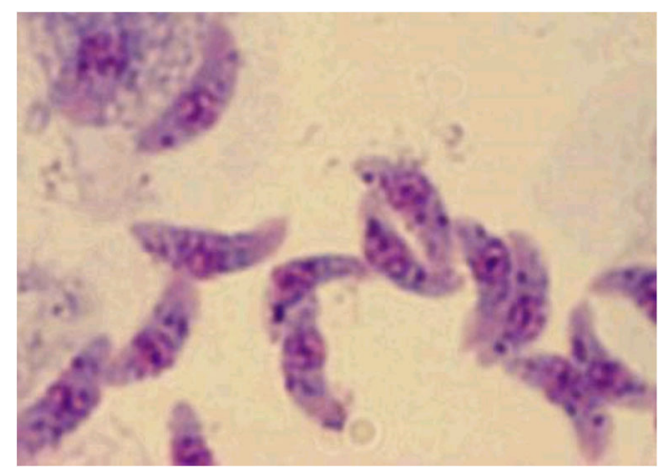

Fig. 1 Sarcocystis bradyzoite by Giemsa staining method (Dob smear). (magnification $\times 1000)$

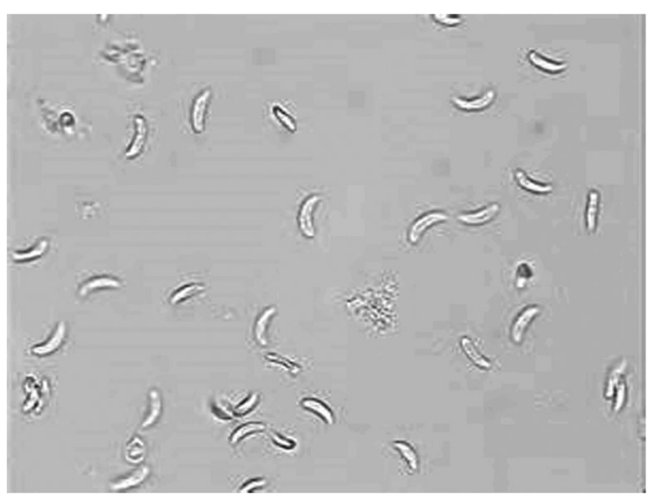

Fig. 2 Sarcocystis bradyzoite by digestion method. (magnification $\times 400)$.

\section{Results}

Our results showed that $16(80 \%)$ of samples were infected with Sarcocystis spp. (Table 1). Based on digestion method, the infection rate in hamburger, sausage, and cocktail were $87.5,83.33$, and $66.66 \%$, respectively. The highest infestation rate was observed in hamburger of plants A and B. Whereas the lowest infection rate was observed in sausage of plant B.

\section{Discussion}

Sarcocystis is an obligatory intercellular parasite in mammals. Several investigations showed that there are considerable infection rate in sheep and cattle that are infected with Sarcocystis spp. in the entire world (Sahl Poulsen and Rune 2014; Fayer et al. 2015; Rosenthal et al. 2012; Mehdizadeh et al. 2014; Meistro et al. 2015; Hajimohammadi et al. 2014a; Jahed Khaniki and Kia 2006; Rahdar and Salehi 2011a; Prayson et al. 2008; Hosseini et al. 2007; Nematollahia et al. 2013). Sarcocystosis is distributed worldwide and more than 150 species of the parasite have been isolated from various domestic and wild animals. Humans are infected with this parasite as intermediate and final host in which the parasite inhabits the muscular and intestinal tract. Studies show that eating of undercooked or infected raw meat or meat products can cause infection in human. Consumption of fast foods in recent years is increasing as American people consume about five billion hamburgers annually (Gabriele et al. 2006).

Many investigations have concerned the prevalence of parasites in foods, especially Sarcocystis spp. In the present study, Sarcocystis was detected in different types of meat products. Several researches have been conducted on the prevalence of Sarcocystis in meat production. Jahed khaniki et al. reported that $6.25 \%$ of hamburger were positive for Sarcocystis cyst (Jahed Khaniki and Kia 2006). Another study has shown that $77.9 \%$ of all tested hamburger were 
Table 1 Infection rate of Sarcocystis spp. in plants A, B, and C

\begin{tabular}{lllllll}
\hline & Plant A & \multicolumn{3}{c}{ Plant B } & \multicolumn{2}{c}{ Plant C } \\
\hline Sample type & No. examined & Infected number (\%) & No. examined & Infected number (\%) & No. examined & Infected number (\%) \\
Hamburger & 4 & $4(100)$ & 1 & $1(100)$ & 3 & $2(66.66)$ \\
Sausage & - & - & 2 & $1(50)$ & 4 & $4(100)$ \\
Cocktail & - & - & 3 & $2(66.66)$ & 3 & $2(66.66)$ \\
\hline
\end{tabular}

infected with Sarcocystis spp. The infection rate in traditional hamburger $(87 \%)$ was significantly higher than the industrial ones (67.8\%) (Hajimohammadi et al. 2014a). Rahdar and Salehi demonstrated that hamburger, sausage, and hotdogs were infected with Sarcocystis in considerable amount in 56, 8, and 20\%, respectively (Rahdar and Salehi 2011a). Prayson et al. found Sarcocystis spp. in two out of eight examined hamburger brands in the USA by using histological method (Prayson et al. 2008). Hosseini et al. reported occurrence of 47.9\% (56 of 117) Sarcocystis infection, by using impression smear assay, in distributed hamburger in Tehran, Iran (Hosseini et al. 2007). Another study in Tabriz announced that the prevalence rate of Sarcocystis spp. in both traditional and industrial hamburger using both impression smear and peptic digestion methods was the same as $56.25 \%$ (Nematollahia et al. 2013). In the present study, we showed that digestion method is better than impression smear method in detection Sarcocystis. This finding agrees with other studies in Iran and the world: Bradyzoites of parasite were observed in $97.14 \%$ of animals' digested muscles in Yazd (Hajimohammadi et al. 2014b). The current study demonstrated that peptic digestion method gave the highest rate $(93.3 \%)$ followed by indirect fluorescent antibody test (IFAT) (88.6\%), squeezing $(81.3 \%)$, and muscle squash (81.2\%) (Latif et al. 1999). Infection rate of Sarcocystis in cattle slaughtered in Shiraz, Iran by digestion impression methods were 100 and 99\% (Shekarforoush et al. 2004).

\section{Conclusion}

Preventing human Sarcocystosis will continue to rely on sanitary approaches to food preparation, but understanding the particular risks and transmission modes in the future will increasingly benefit from improved differential diagnosis based on genetic individuation. Successful methods for rendering specific diagnoses, from either tissue cysts or oocysts, are now available in places (such as China) where raw meat consumption remains prevalent among certain ethnic minorities (Rosenthal et al. 2012). People must be aware of the risk of Sarcocystosis. Thus, for the prevention of human infection, the meat should be frozen or cooked sufficiently before consumption.
Acknowledgements The authors would like to thank Ms. Azami and Ms. Moradi for their help in parasitology laboratory.

\section{Compliance with ethical standards}

Conflict of interest The authors declare that they have no conflict of interest.

Open Access This article is distributed under the terms of the Creative Commons Attribution 4.0 International License (http:// creativecommons.org/licenses/by/4.0/), which permits unrestricted use, distribution, and reproduction in any medium, provided you give appropriate credit to the original author(s) and the source, provide a link to the Creative Commons license, and indicate if changes were made.

\section{References}

Fayer R (2004) Sarcocystis spp. in human infections. Clin Microbiol 17: 894-902

Fayer R, Esposito DH, Dubey JP (2015) Human infections with Sarcocystis species. Clin Microbiol Rev 28:295-311

Fukuyo M, Battsetseg G, Byambaa B (2002) Prevalence of Sarcocystis infection in meat-producing animals in Mongolia. Southeast Asian J Trop Med Public Health 33(3):490-495

Gabriele G, Stefano R, Ottavia G, Eugenio S (2006) Identification and prevalence of Sarcocystis spp. cysts in bovine canned meat. Food Control 17:691-694

Hajimohammadi B, Dehghani A, Moghadam Ahmadi M, Eslami G, Oryan A, Khamesipour A (2014a) Prevalence and species identification of Sarcocystis in raw hamburger distributed in Yazd, Iran using PCR-RFLP. J Food Qual Hazards Control:15-20

Hajimohammadi B, Zohourtabar A, Fattahi Bafghi A, Omidpanah A, Rayati T, Ghasemi A, Sazmand A (2014b) Occurrence and distribution of Sarcocystis parasite isolated from sheep in Yazd Province, Iran. JCHR 3:205-210

Hosseini H, Khaksar R, Shemshadi B (2007) Study on infestation of raw hamburgers to Sarcocystis cyst in Tehran. JFST 4:65-70

Jahed Khaniki GR, Kia EB (2006) Detection of the Sarcocystis cysts from meat supplied for hamburger in Iran by histological method. J Med Sci 6:18-21

Latif BMA, Jk A-D, Mohammed BS, Al-Bayati SM, Al-Amiry AM (1999) Prevalence of Sarcocystis spp. in meat-producing animals in Iraq. Vet Parasitol 84:85-90

Mehdizadeh M, Mousavi SM, Rabiei M, Moradian K, Eskandari S, Abbasi Fesarani M, Rastegar H, Alebouyeh M (2014) Detection of chicken meat adulteration in raw hamburger using polymerase chain reaction. J Food Qual Hazards Control:36-40

Meistro S, Pezzolato M, Botta M, Biglia C, Modesto P, Bozzetta E, Peletto S, Varello K, Richelmi G, Baioni E, Acutis P (2015) Sarcocystis spp. prevalence in bovine minced meat: a histological and molecular study. Ital J food Saf 4:85-87 
Nematollahia A, Khoshkerdar A, Ashrafi Helan J, Shahbazi P, Hassanzadeh P (2013) A study on rate of infestation to sarcocystis cysts in supplied raw hamburgers. J Parasit Dis:1-4

Nourollahi Fard SR, Asghari M, Nouri F (2009) Survey of Sarcocystis infection in slaughtered cattle in Kerman. Iran Trop Anim Health Pro 41:1633-1636

Prayson B, McMahon JT, Prayson RA (2008) Fast food hamburgers: what are we really eating? Ann Diagn Pathol 12:406-409

Rahdar M, Salehi M (2011a) The prevalence of Sarcocystis infection in meat-production by using digestion method in Ahvaz, Iran. Jundishapur J Microbiol 4:295-299
Rahdar M, Salehi M (2011b) The prevalence of Sarcocystis infection in meat-production by using digestion method in Ahvaz. Iran Jundishapur J Microbiol 4(4):295-299

Rosenthal B, Yang Z, Yuan L (2012) Sarcocystis spp. In: Lucy J, Robertson and Huw, Smith V (eds) Foodborne Protozoan Parasites, USDA: NOVA Science Publishers. p 151-166

Sahl Poulsen C, Rune Stensvold C.2014 Human Sarcocystosis: Current Status on Epidemiology and Diagnosis. J Clin Microbiol. doi:10. 1128/JCM.00955-14

Shekarforoush SS, Razavi SM, Ahmadi H, Sarihi K (2004) Study on prevalence of Sarcocystis in slaughtered cattle in Shiraz. J Vet Res 59:33-37 